\title{
Effects of Caffeine Consumption on Autologous Full-Thickness Skin Graft Healing in an Animal Model
}

Tommy Supit ${ }^{1,2, \odot}$ Neni Susilaningsih ${ }^{2,3}$ Awal Prasetyo ${ }^{2,4}$ Najatullah

\author{
1Department of Surgery, Faculty of Medicine Diponegoro \\ University, Dr. Kariadi General Hospital, Semarang, Jawa Tengah, \\ Indonesia \\ 2Department of Biomedical Science, Faculty of Medicine \\ Diponegoro University, Semarang, Jawa Tengah, Indonesia \\ ${ }^{3}$ Department of Histology, Faculty of Medicine Diponegoro \\ University, Semarang, Jawa Tengah, Indonesia \\ ${ }^{4}$ Department of Anatomical Pathology, Faculty of Medicine \\ Diponegoro University, Dr. Kariadi General Hospital, Semarang, \\ Jawa Tengah, Indonesia \\ ${ }^{5}$ Department of Plastic Surgery, Faculty of Medicine Diponegoro \\ University, Dr. Kariadi General Hospital, Semarang, Jawa Tengah, \\ Indonesia
}

Indian J Plast Surg 2021;54:314-320.

\author{
Address for correspondence Tommy Supit, MD, MBiomed, \\ BMedSc(Hons), Department of General Surgery, Faculty of Medicine \\ Diponegoro University, Dr. Kariadi General Hospital Jl. Dr. Sutomo \\ No.16, Randusari, Semarang 50244, Jawa Tengah, Indonesia \\ (e-mail: tommy.supit@gmail.com).
}

\author{
Abstract \\ Keywords \\ - caffeine \\ - graft \\ - autologous \\ - full-thickness skin \\ graft \\ - wound \\ - healing
}

Background There exists contradictory evidence that states both the beneficial and deleterious effects of caffeine on wound healing. The general population might unknowingly consume caffeine that negatively affects wound healing. The main objective of this study is to investigate the effect of daily caffeine consumption on wound healing, specifically full-thickness skin graft (FTSG).

Methods Forty Sprague-Dawley rats were randomized into four groups of equal size: control-dose (CD), low-dose (LD), medium-dose (MD), and high-dose (HD) caffeine groups. After autologous FTSG, all subjects in the intervention group were given daily pure caffeine gavage. The FTSG was explanted 7 days posttransplant. The graft viability, secondary contraction, and adherence were evaluated macroscopically, while fibroblast and collagen deposition was analyzed microscopically with hematoxylin eosin stain.

Results The least graft viability $(72.8 \pm 20.7 \%$, clinical wound assessment scale [CWAS] 2.4), highest secondary contraction (11.4 $\pm 10.5 \%)$, and fibroblast count (331.8 \pm 88.6 cells $/ 5$ high power fields) were observed in the MD group. More collagen synthesis was observed in subjects who consumed caffeine. The level of secondary contraction, fibroblast count as well as graft viability and collagen synthesis were positively correlated.

Conclusions Daily consumption of caffeine impairs graft viability when given in medium dose and increases collagen synthesis, irrespective of dosage. This study was in experimental rats; the results are not directly translatable to humans. published online

September 16, 2021
DOI https://doi.org/

$10.1055 / \mathrm{s}-0041-1734573$

ISSN 0970-0358
(C) 2021. Association of Plastic Surgeons of India.

This is an open access article published by Thieme under the terms of the Creative Commons Attribution-NonDerivative-NonCommercial-License, permitting copying and reproduction so long as the original work is given appropriate credit. Contents may not be used for commercial purposes, or adapted, remixed, transformed or built upon. (https://creativecommons.org/licenses/by-nc-nd/4.0/).

Thieme Medical and Scientific Publishers Pvt. Ltd. A-12, 2nd Floor, Sector 2, Noida-201301 UP, India 


\section{Introduction}

Continuous efforts are being made to increase the survivability of skin grafts, from improving surgical techniques to using advanced dressings. ${ }^{1}$ Intrinsic factors such as comorbidities, smoking, or nutritional status play an equally important role in successful grafting. ${ }^{2}$ Major interests are rising in this field, especially with regard to the role of nutrition and strategic dietary supplements on wound healing. Caffeine is present in widely consumed beverages such as coffee, tea, and carbonated drinks, and some patients undergoing surgery are bound to be daily consumers of caffeine. ${ }^{3}$

The antioxidative and antibacterial properties of caffeine have the potential to promote wound healing. ${ }^{4}$ On the other hand, caffeine was shown to impair epithelialization, contradicting the hypothetical benefits. ${ }^{5}$ The healing-impairment mechanisms of caffeine were thought to be primarily caused by its action as a nonselective adenosine receptor antagonist, impairing cell proliferation, differentiation, migration, and angiogenesis that ultimately inhibit physiological healing. ${ }^{6}$ The other mechanism by which caffeine might affect wound healing is through its anti-inflammatory action, which remains to be elucidated whether it is beneficial for reducing scar tissue formation or impairing graft intake. ${ }^{7}$ There is insufficient knowledge on the effect of caffeine consumption on cutaneous wound healing, necessitating studies that explore specific mechanisms and the impact of caffeine as an independent risk factor for wound healing. Until then, it is unjustified to recommend patients to alter their caffeine consumption habit or to supplement them with one. The objective of this study is to investigate the effects of different doses of daily caffeine consumption on full-thickness skin graft (FTSG) healing in experimental rats: graft viability, adherence, secondary contraction, fibroblast proliferation, and collagen synthesis.

\section{Materials and Methods}

Ethical clearance was obtained from governing ethical committees, and all animals were housed in a facility compliant in accordance with the National Health and Medical Research guidelines (No. 85/EC/H/FK-RSDK/VII/2018; No 1084/UN7.8/PP/2018).

\section{Experimental Subjects}

Forty male Sprague-Dawley rats aged between 13 to 15 weeks were randomized into four experimental groups of equal size ( $n=10$ rats/group): control-dose (CD), low-dose (LD), medium-dose (MD), and high-dose (HD) caffeine groups. All rats were provided with standard rat chow and clean water ad libitum, and kept under controlled temperature at $20{ }^{\circ} \mathrm{C}$ with a 12-hour day-night cycle. All subjects were acclimatized for 7 days before the start of the intervention. The study was conducted in May and lasted until June 2018.

\section{Surgical Procedure}

General anesthesia was performed using intraperitoneal injection of ketamine-xylazine (ketamine $80 \mathrm{mg} / \mathrm{kg}$, xylazine
$10 \mathrm{mg} / \mathrm{kg}$ ). After the skin preparation, a square-shaped FTSG harvest site measuring $1.5 \mathrm{~cm} \times 1.5 \mathrm{~cm}$ was marked on the right hemithorax. Subcutaneous fat, panniculus carnosus, and loose connective tissue were cleared from the graft and wound bed. The harvested FTSG was rotated $180^{\circ}$ from its original orientation, placed back to the same donor site ( - Fig. 1 A, B), and sutured with polyvinylidene fluoride $4 / 0$ threads (-Fig. 1 C-F). One investigator performed the surgery, documentation, and postoperative care and monitoring.

\section{Caffeine Gavage}

The different dosages of caffeine given to the rats were allometrically scaled from human equivalent doses: LD 3, MD 6, and HD $9 \mathrm{mg} / \mathrm{kg}$ body weight into rat doses: LD 19, MD 37, and HD $56 \mathrm{mg} / \mathrm{kg}^{8.9}$ The caffeine anhydrous powder (Purebulk, US) was dissolved in clean drinking water, creating a final solution concentration of $7.4 \mathrm{mg} / \mathrm{mL}$. Control rats were gavaged drinking water. The rats were returned into the cage and observed for at least 10 minutes for any signs of regurgitation after gavage.

\section{Macroscopic Evaluation}

All rats were euthanized 7 days postsurgery with cervical dislocation following general anesthesia using ketamine-xylazine. Parameters measured included graft viability, secondary contraction, and adherence. Evaluation of graft viability and secondary contraction was based on photographs taken directly postgraft (day 0 ) and during necropsy (day 7). Clinical photographs were taken $15 \mathrm{~cm}$ above the object, using Samsung 16-megapixel camera (1428 $\times$ 1667 pixels, focal length 3.6, exposure time 1/868). Areas of dark-ischemic tissue or with doubtful viability of day-7 FTSG were traced manually and then divided by its total surface area, expressed in percentage of graft surface area. Quantification was performed using ImageJ Version 1.51 (USA National Institute of Health) by manually tracing the borders of FTSG. Evaluation of graft secondary contraction was conducted in a similar fashion. Graft adherence to the wound bed was evaluated by pulling the graft upward from the wound bed and inspection of soft-tissue attachment on the posterior side of the explants. Quantified results of FTSG viability were then graded using the clinical wound assessment scale (CWAS) (-Table 1). ${ }^{10}$ Two blinded investigators performed the macroscopic evaluation.

\section{Microscopic Evaluation}

During necropsy, the FTSG was resected along with $0.5 \mathrm{~cm}$ surrounding tissue involving the skin and underlying thoracic muscle, creating an explanted tissue measuring $2 \mathrm{~cm}$ $\times 2 \mathrm{~cm}$. One blinded board-certified pathologist quantified the number of fibroblasts from five high power fields per sample at $\times 40$ magnification. Collagen deposition analysis was performed using ImageJ software by threshold analyses of microscopic photographs captured using Leica ICC50 HD digital microscope (LAS EZ software, dimension $2048 \times 1536$ pixels). The threshold was adjusted until only the collagen was shaded and the edges of granulation tissue 
A.
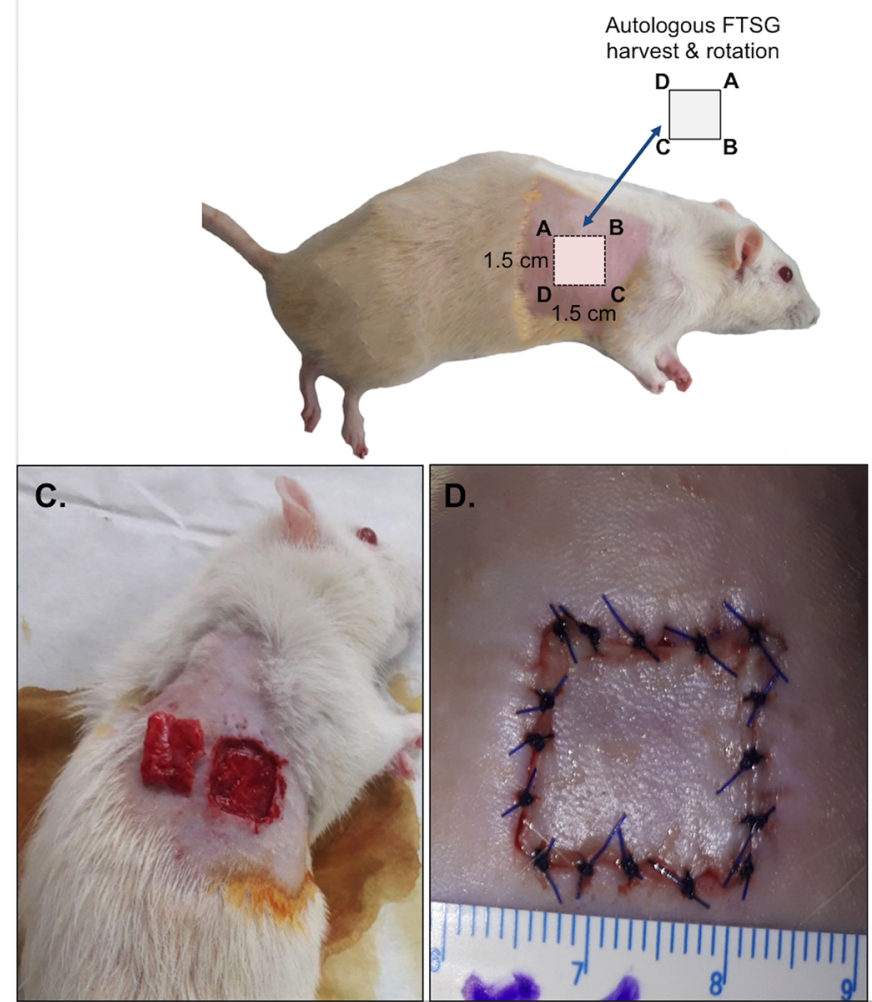

B.
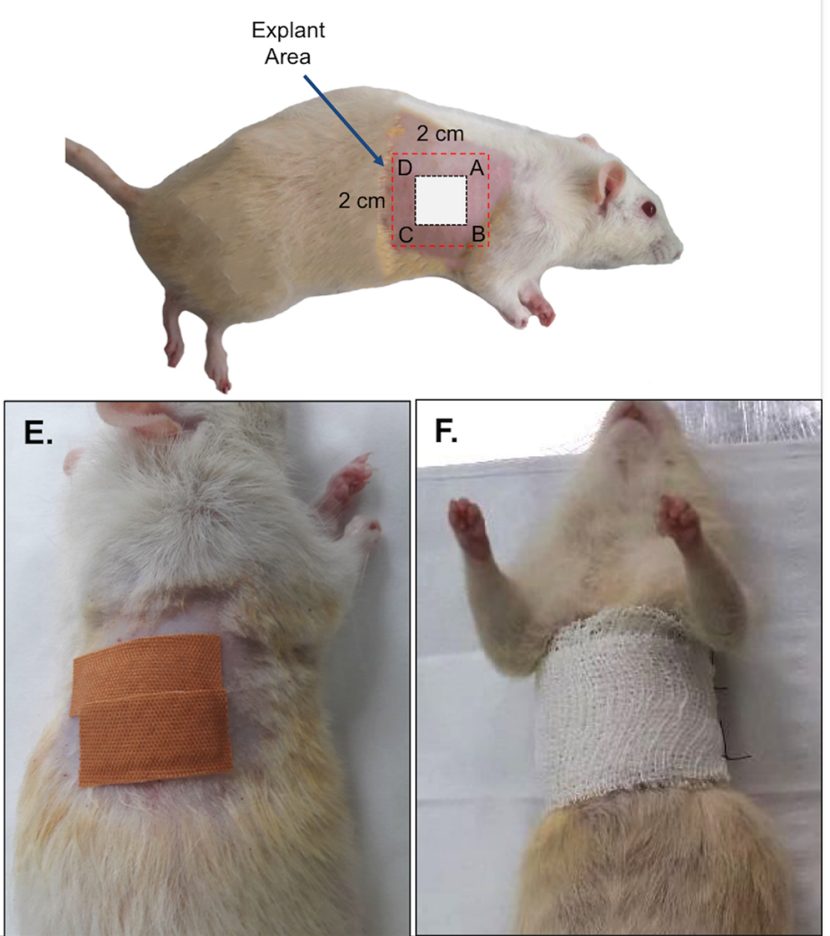

Fig. 1 (A-F). Illustrated photographs of autologous FTSG technique. Harvest and re-transplantation of full-thickness skin graft (FTSG) on to the donor site (A, C) that was explanted after 7 days (B). Perioperative photographs showing graft suturing and bandaging (D-F).

Table 1 CWAS.

\begin{tabular}{|l|l|l|}
\hline Grade & Graft viability & Graft adherence \\
\hline 0 & Fully viable, no dislocation & Full graft loss \\
\hline 1 & Fully viable, $5-10 \%$ dislocation & $20 \%$ adherent \\
\hline 2 & $>10 \%$ not viable & $50 \%$ adherent \\
\hline 3 & $>20 \%$ not viable & $75 \%$ adherent \\
\hline 4 & $>50 \%$ not viable & $90 \%$ adherent \\
\hline 5 & Complete graft loss & $\begin{array}{l}\text { Full graft } \\
\text { adherence }\end{array}$ \\
\hline
\end{tabular}

Abbreviation: CWAS, clinical wound assessment scale.

traced manually. The index of collagen synthesis was defined as the ratio of surface area of new collagen to the surface area of the granulation tissue.

\section{Statistical Analysis}

Statistical analysis was performed using SPSS Version 22 (IBM Corporation, USA). Data were tested for normality using the Shapiro-Wilk test. The rat's body weight, graft contraction, adherence, viability, and fibroblast count were analyzed using the Kruskal-Wallis test, followed by Dunn's multiple comparison posthoc tests. Collagen deposition was analyzed using one-way ANOVA test, followed by Bonferroni posthoc tests. Spearman's correlation analysis was performed to find significant correlation between variables. Values of $p<$ 0.05 were considered to be statistically significant. Data was expressed as mean \pm standard deviation (SD) unless stated otherwise.

\section{Results}

\section{Macroscopic Evaluation}

A variable degree of elastic and nonadhesive bandage damage with undisturbed FTSG was observed in 10 rats, which was reapplied without any manipulation of the FTSG. The conditions of these subjects were observed to be generally the same with the rest of group, showing normal activity and robust movements. No local or systemic signs of infection were observed, and all rats survived the experiment until the planned time of explant. The necrotic or parts of compromised viability were mainly observed at the periphery of FTSF near the sutures ( - Fig. 2 B). The least amount of viable FTSG was found in MD (CWAS $2.4 \pm 1.1 ; 72.8 \pm 20.7 \%$ viable tissue), followed by HD $(1.4 \pm 0.5 ; 85.1 \pm 9.2 \%)$, CD (1.0 $\pm 0.7 ; 92.8 \pm 6.2 \%)$, and $\mathrm{LD}(1.0 \pm 0.0 ; 92.8 \pm 3.5)$. All FTSGs underwent secondary contraction with CD $9.7 \pm 5.6 \%$, LD 8.2 $\pm 1.3 \%$, MD $11.4 \pm 10.5 \%$ and HD $5.4 \pm 6.2 \%$ reduction of graft surface area (-Fig. 2 B). Most of the FTSGs adhered to the wound bed adequately with a combination of thick fibrous and loose-fatty connective tissue ( - Fig. 2 D). Only areas of the graft that was not attached to any underlying connective tissue during explant were considered to be nonadherent. The CWAS scores of adherences were similar for all groups: CD $4.0 \pm 1.4$, LD $4.2 \pm 0.8$, MD $4.0 \pm 1.0$, and HD $4.2 \pm 0.9$ with no statistically significant difference.

\section{Microscopic Evaluation}

Fibroblasts were mainly identified within the newly formed connective tissue among the strands of new collagen 


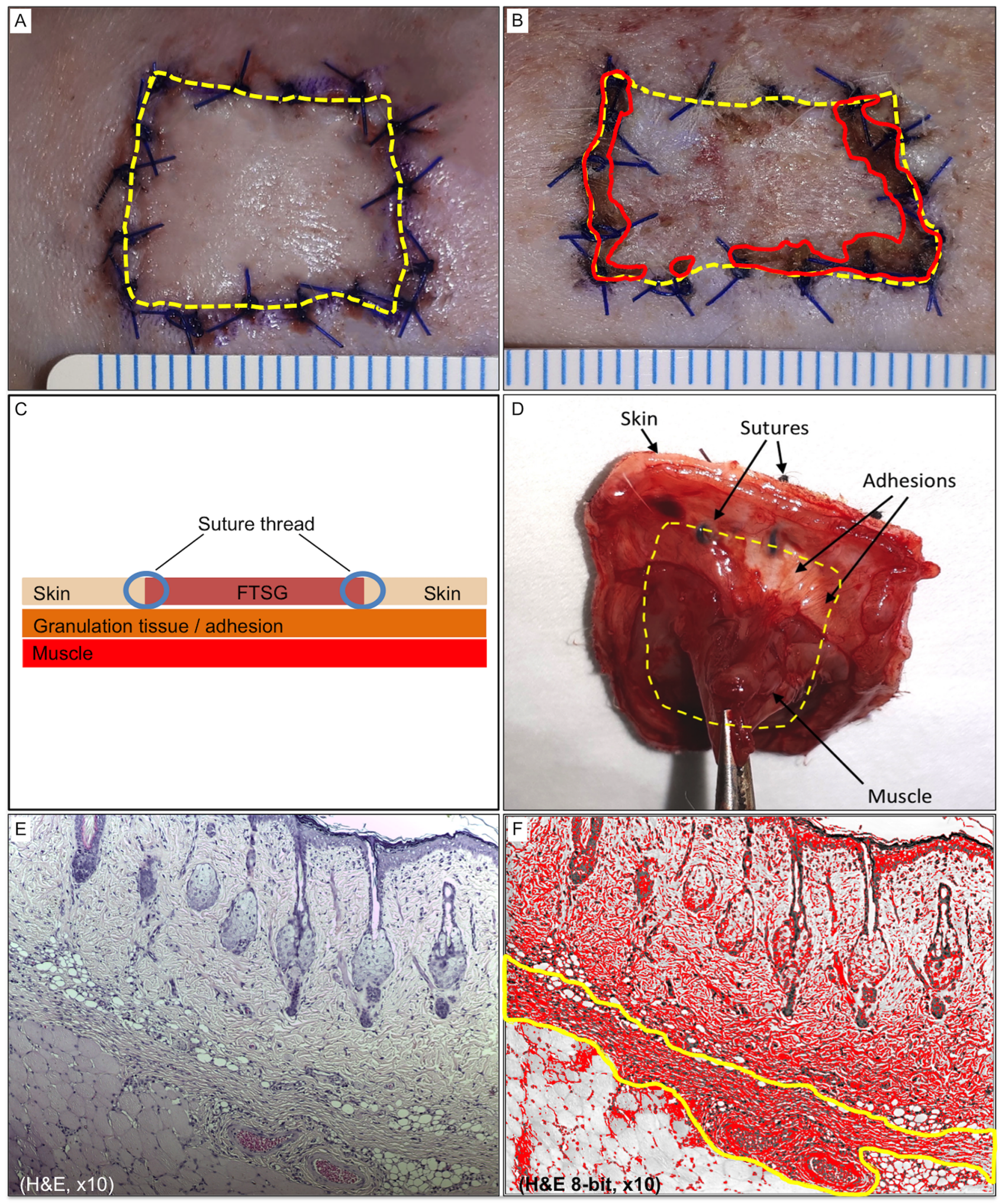

Fig. 2 (A-F). Tissue analysis technique. Manual tracing for quantifying the full-thickness skin graft (FTSG) surface area and nonviable region (A, B). Schematic illustration of the cross-sectional view (C) and clinical photograph of posteroanterior view of the explanted graft (D). Cross-section of H\&E stain explant showing the three distinct tissue layers (E) and after threshold analysis of the same image selecting the collagen layers sandwiched between the skin graft and muscle tissue (F).

(-Fig. 2 E). The highest fibroblast count was observed in MD (331.8 \pm 88.6 cells/5HPF), followed by CD (319.0 \pm 90.9 cells/5HPF), LD (272.8 \pm 69.8 cells/5HPF), and HD (237.8 \pm 50.1 cells $/ 5 \mathrm{HPF}$ ) ( - Fig. 3 ). Compared with the mature collagen network within the FTSG, newly synthesized collagen was identified by its lighter pink shades with straighter and thinner fibers that run parallel with the underlying muscle layer. There were statistically significant differences of 

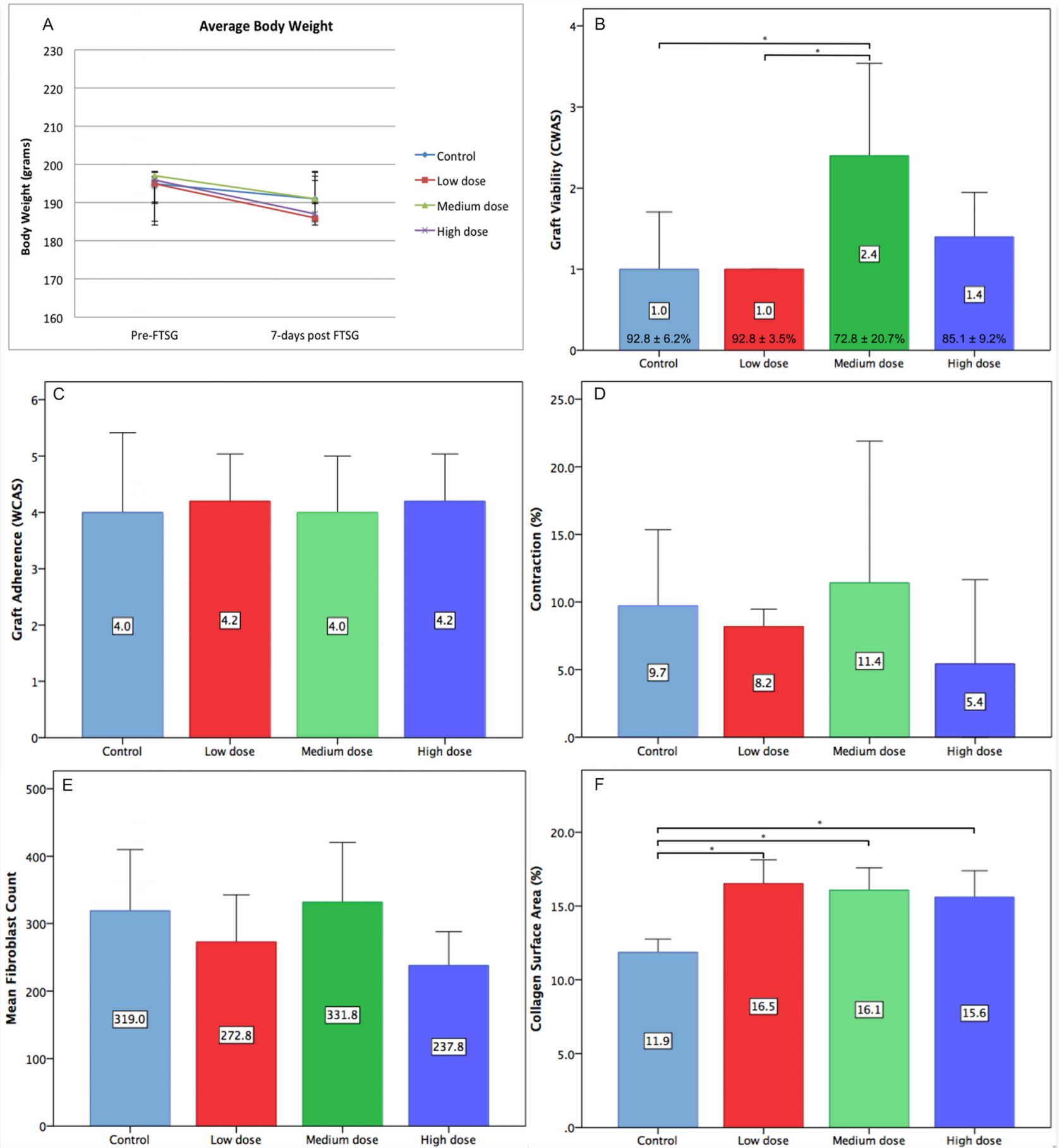

Fig. 3 (A-F). Results. Data were expressed as mean \pm standard deviation (SD) unless stated otherwise, $n=10$ rats $/$ group, ${ }^{*} p<0.05$. The average subjects' body weight pre- and post- full-thickness skin graft (FTSG) (A). Graft viability graded with clinical wound assessment scale (CWAS), percentage of graft viability presented at the bottom of the bars (B). Graft adherence graded with CWAS (C). Graft contraction presented in percentage of surface area reduction (D). Fibroblast count presented in cells/five HPF (E). Collagen surface area presented as percentage of surface area $(\mathbf{F})$.

collagen deposition level between the $\mathrm{CD}(11.9 \pm 0.9 \%)$ and the rest of the intervention group. There were moderate positive correlations between contraction and fibroblast count (0.31) as well as between viability and collagen (0.48). A moderate negative correlation was found between adherence and fibroblast $(-0.40)$ (-Table 2).

\section{Discussion}

\section{Graft Viability and Adherence}

The healing process of FTSG begins with the imbibition, followed by inosculation, revascularization, and reinnervation. ${ }^{11}$ Data acquired from this study can be extrapolated to 
Table 2 Spearman's correlation between variables

\begin{tabular}{|l|l|l|l|l|l|}
\hline & $\mathbf{1}$ & $\mathbf{2}$ & $\mathbf{3}$ & $\mathbf{4}$ & $\mathbf{5}$ \\
\hline 1. Contraction & & & & & \\
\hline 2. Adherence & 0.25 & & & & \\
\hline 3. Viability & 0.23 & 0.23 & & & \\
\hline 4. Fibroblast & 0.31 & -0.40 & -0.14 & & \\
\hline 5. Collagen & -0.02 & 0.15 & 0.48 & -0.30 & \\
\hline
\end{tabular}

split-thickness skin graft (STSG) since both FTSG and STSG underwent a similar integration process. The worse viability was observed in MD group with an average of $72.8 \pm 20.7 \%$, which was considerably higher compared with two other studies. Takabayashi et al found the average viable graft surface area 10 days posttransplant in the control group was 31.1 $\pm 6.6 \%(n=12) .{ }^{12}$ In another study conducted by Zhang et al, the viability of $3 \times 3 \mathrm{~cm}$ FTSG was reduced to only $22.5 \pm 7.7 \%$ $(n=8)$ on day 5 posttransplant. ${ }^{13}$ In the first study, extracellular edema created by cutaneous injection of saline might have interfered the imbibition process, impairing the transfer of nutrition.

The whole layer skin-to-skin sutures that lie between the muscle and graft might have acted as a physical barrier to the plasmatic imbibition ( - Fig. 2). Graft tissue within the suture knots might have also been $\mathbf{C}$ strangulated, preventing vascular anastomosis and angiogenesis, and resulting in nonviable tissue that was observed mostly at the periphery of the graft. Thus, compromised viability was mostly observed on the edges of the FTSG where the sutures were. The intradermal suturing technique might be a solution to this problem, since no suture material will be placed between the graft and the nourishing wound bed.

The rat skin is not analogous to human skin, because of the presence of subcutaneous tissue, which is called the panniculus carnosus and contributes to skin healing by contraction, collagen formation, and capillary remodeling. ${ }^{14,15}$ With that in light, panniculus carnosus was considered as a confounding factor and thus removed in the experiment to better mimic the human condition. In our study, the FTSG adherence was observed to be satisfactory and almost consistent in all experimental groups, showing no evidence that caffeine consumption impairs this function. Adherence depends on the architecture of collagen with other ECM as well as the newly formed blood vessels. ${ }^{16}$ Hypothetically, caffeine impairs adherence through its action as adenosine receptor antagonist that prevents fibroblast action and endothelial cell proliferation.

\section{Fibroblast, Collagen Synthesis and Secondary Graft Contraction}

Caffeine was shown to inhibit transforming growth factor $\beta$ (TGF- $\beta$ ) activation in epithelial cells in ex vivo studies involving human lung and liver tissue. ${ }^{17,18}$ It can be hypothesized that caffeine may reduce secondary contraction by the downregulation of TGF- $\beta$, a key player in the activation of fibroblast. In an ex vivo using human fibroblast culture, caffeine inhibited collagen synthesis by inhibiting the action of prolidase activity, other than reducing the proliferative capacity of skin fibroblast. ${ }^{19}$ In this study, the fibroblast count interestingly mimics the trend of secondary contraction; the highest fibroblast number was found in MD group, followed by $\mathrm{CD}, \mathrm{LD}$, and HD.

Spearman's rank test found a medium positive correlation (0.31) between the two variables. In terms of collagen biosynthesis, this experiment demonstrated that a daily dose of caffeine positively affects its production. More collagen was observed in rats who received caffeine independent of doses, which contrasted to another study where exposure of fibroblasts to different concentrations of caffeine led to inhibition of collagen synthesis by $48.1 \% \pm 2.4 \%(n=3)$ in low dose $(1 \mathrm{mM}), 72.9 \% \pm 3.6 \%(n=3)$ in medium dose $(2 \mathrm{mM})$, and $92.1 \% \pm 3.8 \%(n=3)$ in high hose $(5 \mathrm{mM}) .{ }^{19}$ The distinct results can be caused by an inherent methodological difference between the two experiments; our study is in vivo where the caffeine was given orally to live rats, while the other was ex vivo. Orally consumed caffeine is metabolized in the liver and its plasma concentration is greatly affected by the first-order kinetics that will affect the amount of caffeine that will reach the granulation tissue. ${ }^{20}$

The amount of new collagen is not proportional with the number of fibroblasts within the granulation tissue, which suggests that collagen synthesis is not affected by the quantity of fibroblast per se but more to the quality of their activity. In this case, active fibroblasts may have reverted back to its inactive form, fibrocytes, after synthesizing collagen and other extracellular matrix (ECM) components. ${ }^{21}$ The exact mechanism by which oral caffeine consumption affects collagen synthesis but not fibroblast proliferation is still unknown and should be looked into further.

Secondary contraction is primarily mediated by myofibroblasts' interaction with ECM and collagen. All FTSGs underwent secondary contraction in this study, with the highest contraction observed in MD (11.4 $\pm 10.5 \%)$ and the lowest in HD $(5.4 \pm 6.2 \%)$. Tuncali et al found the exact size FTSG was reduced to $91.0 \pm 2.8 \%$ and Kamran et al' FTSG reduced to $88.3 \pm 15.0 \%$ of its original surface area at 7 days posttransplant. ${ }^{22,23}$ It can be inferred that caffeine does not affect secondary contraction. Another factor that might have contributed to the degree of secondary contraction is the suture depth. In this experiment, the FTSG was sutured to the skin of wound edges, excluding the underlying muscle. Suturing the FTSG to the muscle layer will provide greater tissue fixation, resisting dermal contraction on the transverse plane. 
However, this step might cause pain and discomfort for the rat; hence, it was not performed in this study. Aside from the depth, the suturing technique can affect secondary contraction because tension between two wound edges can be adjusted individually with interrupted sutures, which is not possible with continuous technique.

The authors identified several study limitations. The microscopic analysis has a relatively high degree of observer subjectivity and variability between measurements. Collagen deposition analysis in this study was performed based on its quantity (surface area) per se and was limited to a two-dimensional plane. The network of collagen and its complex interaction with other ECM components is better appreciated in three-dimensional plane using hydroxyproline quantification. Better visualization of newly synthesized collagen fibers and fibroblast activity can also be achieved by using immunohistochemistry, for example, antibody GD3A12 or radioactive labeling assay. As with every animal experimental study, the results of this investigation are not directly translatable to human. The limited sample size could have affected the results, which do not show a linear dose-response relationship. The investigation of FTSG healing in this study was limited to the acute phase. Future studies should involve more dosing points to better appreciate the effect of caffeine on complete integration process of FTSG, evaluating the end graft size, quality of skin, hair growth, and innervation.

In conclusion, daily consumption of caffeine affects FTSG healing in Sprague-Dawley rats. The least amount of graft viability, greatest secondary contraction, and highest amount of fibroblast were observed in subjects that consumed caffeine in medium dose. Increased collagen synthesis was observed in all subjects who received caffeine. Moderate positive correlations were observed between the number of fibroblasts and the degree of secondary contraction as well as between FTSG viability and collagen synthesis. To our knowledge, this is the first study that investigated the effect of caffeine consumption on FTSG healing.

\section{Financial Disclosure}

None.

\section{Conflict of Interest}

None declared.

\section{References}

1 Ogawa R, Hyakusoku H, Ono S. Useful tips for successful skin grafting. J Nippon Med Sch 2007;74(6):386-392

2 Cereda E, Neyens JCL, Caccialanza R, Rondanelli $M$, Schols JMGA. Efficacy of a disease-specific nutritional support for pressure ulcer healing: A systematic review and meta-analysis. J Nutr Health Aging 2017;21(6):655-661

3 Poole R, Kennedy OJ, Roderick P, Fallowfield JA, Hayes PC, Parkes J. Coffee consumption and health: umbrella review of meta-analyses of multiple health outcomes. BMJ 2017;359:j5024
4 Almeida AA, Farah A, Silva DAM, Nunan EA, Glória MBA. Antibacterial activity of coffee extracts and selected coffee chemical compounds against enterobacteria. J Agric Food Chem 2006;54(23):8738-8743

5 Ojeh N, Stojadinovic O, Pastar I, Sawaya A, Yin N, Tomic-Canic M. The effects of caffeine on wound healing. Int Wound J 2016;13(5):605-613

6 Feoktistov I, Biaggioni I, Cronstein BN. Adenosine receptors in wound healing, fibrosis and angiogenesis. Handb Exp Pharmacol 2009;( 193):383-397

7 Horrigan LA, Kelly JP, Connor TJ. Immunomodulatory effects of caffeine: friend or foe? Pharmacol Ther 2006;111(3):877-892

8 Spriet LL. Exercise and sport performance with low doses of caffeine. Sports Med 2014;44(Suppl 2):S175-S184

9 Nair $A B$, Jacob S. A simple practice guide for dose conversion between animals and human. J Basic Clin Pharm 2016;7(2):27-31

10 Branski LK, Mittermayr R, Herndon DN, et al. A porcine model of full-thickness burn, excision and skin autografting. Burns 2008;34(8):1119-1127

11 Šmahel J. Biology of the stage of plasmatic imbibition. Br J Plast Surg 1971;24(2):140-143

12 Takabayashi Y, Ishihara M, Kuwabara M, et al. Improved survival of full-thickness skin graft with low-molecular weight heparin-protamine micro/nanoparticles including platelet-rich plasma. Ann Plast Surg 2017;78(5):562-568

13 Zhang F, Oswald TM, Lin L, Wang S, Lin S, Lineaweaver WC. Improvement of full-thickness skin graft survival by application of vascular endothelial growth factor in rats. Ann Plast Surg 2008;60(5):589-593

14 Kawamata S, Ozawa J, Hashimoto M, Kurose T, Shinohara H. Structure of the rat subcutaneous connective tissue in relation to its sliding mechanism. Arch Histol Cytol 2003;66(3):273-279

15 Naldaiz-Gastesi N, Bahri OA, López de Munain A, McCullagh KJA, Izeta A. The panniculus carnosus muscle: an evolutionary enigma at the intersection of distinct research fields. J Anat 2018;233(3):275-288

16 Tavis MJ, Thornton JW, Harney JH, Danet RT, Woodroof EA, Bartlett RH. Mechanism of skin graft adherence: collagen, elastin, and fibrin interactions. Surg Forum 1977;28:522-524

17 Arauz J, Zarco N, Segovia J, Shibayama M, Tsutsumi V, Muriel P. Caffeine prevents experimental liver fibrosis by blocking the expression of TGF- $\beta$. Eur J Gastroenterol Hepatol 2014;26(2):164-173

18 Tatler AL, Barnes J, Habgood A, Goodwin A, McAnulty RJ, Jenkins G. Caffeine inhibits TGF $\beta$ activation in epithelial cells, interrupts fibroblast responses to TGF $\beta$, and reduces established fibrosis in ex vivo precision-cut lung slices. Thorax 2016;71(6):565-567

19 Donejko M, Przylipiak A, Rysiak E, Głuszuk K, Surażyński A. Influence of caffeine and hyaluronic acid on collagen biosynthesis in human skin fibroblasts. Drug Des Devel Ther 2014;8:1923-1928

20 Burdan F, Pharmacology of Caffeine. In: Coffee in Health and Disease Prevention. Elsevier; 2015:823-829

21 Grieb G, Steffens G, Pallua N, Bernhagen J, Bucala R. Circulating fibrocytes-biology and mechanisms in wound healing and scar formation. Int Rev Cell Mol Biol 2011. 291:1-19

22 Tuncali D, Yavuz N, Cigsar B, Gokrem S, Aslan G. Effect of full-thickness skin graft initial dimension on secondary wound contraction: experimental study in rats. Dermatol Surg 2005;31(5):542-545

23 Kamran A, Javad FM, Sahram F, Jaber MS. A comparison of survival and secondary contraction in expanded versus conventional full-thickness skin grafts: an experimental study in rats. Eplasty 2012;12:e20 